\title{
Optimum Frequency Allocation Mechanism of TV White Spaces by using Interval Graph
}

\author{
Meghalatha.CK, B.Seetha Ramanjaneyulu
}

\begin{abstract}
The unused frequencies of terrestrial TV are being explored in the recent years, to satisfy the bandwidth demands of ever-increasing wireless communication systems. Allocating these unused TV frequencies is often a challenging task. Allocations that fulfil the user requirements at various instants, while maximizing the utilization of available TV frequencies is the one that is desired. In this paper, interval-graph method is implemented to identify the optimum number of channels needed for the given demand of bandwidths. Simulations are carried out by using GLPK 4.65 solver. Results show the appropriate number of channels required or conversely the reduction of data rates to individual users based on the available bandwidths.
\end{abstract}

Keywords: Cognitive Radio, Interval graph, Subcarriers, LTE, TVWS.

\section{INTRODUCTION}

Rapidly-growing number of wireless devices lead to the spectrum scarcity which is a severe problem. To overcome this scarcity problem, Cognitive radio (CR) was proposed. It senses the unused Spectrum holes and accesses the spectrum opportunistically. CR proposed by Joseph Mitola in [1], makes use of an intelligent transceiver which can detect the unused spectrum holes. In this context, the licensed users are called primary users (PUs) and the unlicensed users are called Secondary users (SUs). Unused spectrum holes are available in terrestrial TV frequencies, military frequency allocations and frequencies allocated for navigation purposes. Of these, unused TV frequencies are the most preferred ones to be used in opportunistic manner, because of simpler regulations and availability in large quantities. These TV white spaces can be used for many wireless communication needs including the bandwidthhungry systems like the mobile data transmissions.

Appropriate care needs to be taken while accessing the spectrum opportunistically, because it may lead to interference if two or more devices access the same channel at the same time. As TVWS frequencies are geographicspecific, the allocations made are usually applicable to the given geographic region. As orthogonal frequency division multiplexing (OFDM) is being used in many recent wireless technologies, the allocation of TVWS frequencies need to be made in line with the OFDM mechanism and its sub-carrier based allocations.
In this work also, the allocations are considered in this manner. Also, as LTE based cellular systems are popular at present, the subcarrier bandwidths and resource blocks used in LTE are considered here for discussion. This helps in proposing appropriately the TVWS allocations for mobile data transmission needs [1].

In this work, it is proposed at first, to identify the number of channels needed to support the data transmission requests of the users. If those many channels are available in TVWS frequencies, they can be allocated to the users. Here Interval graph is used to find the optimum number of frequencies that are required to satisfy the user demands at given instant.

Often it may be needed to work from the other direction, like how many users can be offered with frequency allocations, at the given instant. This is important because available frequencies are not in the control of users and so it may be needed to find out how many user requests can be fulfilled with available channels at that time. If it is not required to allot a complete channel to each requested user, then the more users can be accommodated by allotting part of the channel bandwidth to each user. This can be based on subcarrier mechanism, where the channel is considered as sum of multiple subcarriers, and the requesting user can be offered a set of subcarriers from the total subcarriers of a channel.

So, a model that deals with assessment of user needs for channels, based on their arrival rates and channel occupancy duration, is considered, at first. Extension of it by including the option of variable data rate requirement scenario for different users, is considered next. Finally, a practical implementation of the above in terms of subcarrier allocation is considered.

Section-2 of the paper describes the importance of Cognitive radio and LTE. Section-3 explains the significance of Interval graph to obtain

\footnotetext{
Revised Manuscript Received on December 15, 2019

Meghalatha.CK, ECE Department VFSTR University Vadlamudi, Guntur, AP,INDIA. megaanirvesh5@gmail.com

B.Seetha Ramanjaneyulu, ECE Department VFSTR University Vadlamudi, Guntur, AP,INDIA. ramanbs@gmail.com
} 


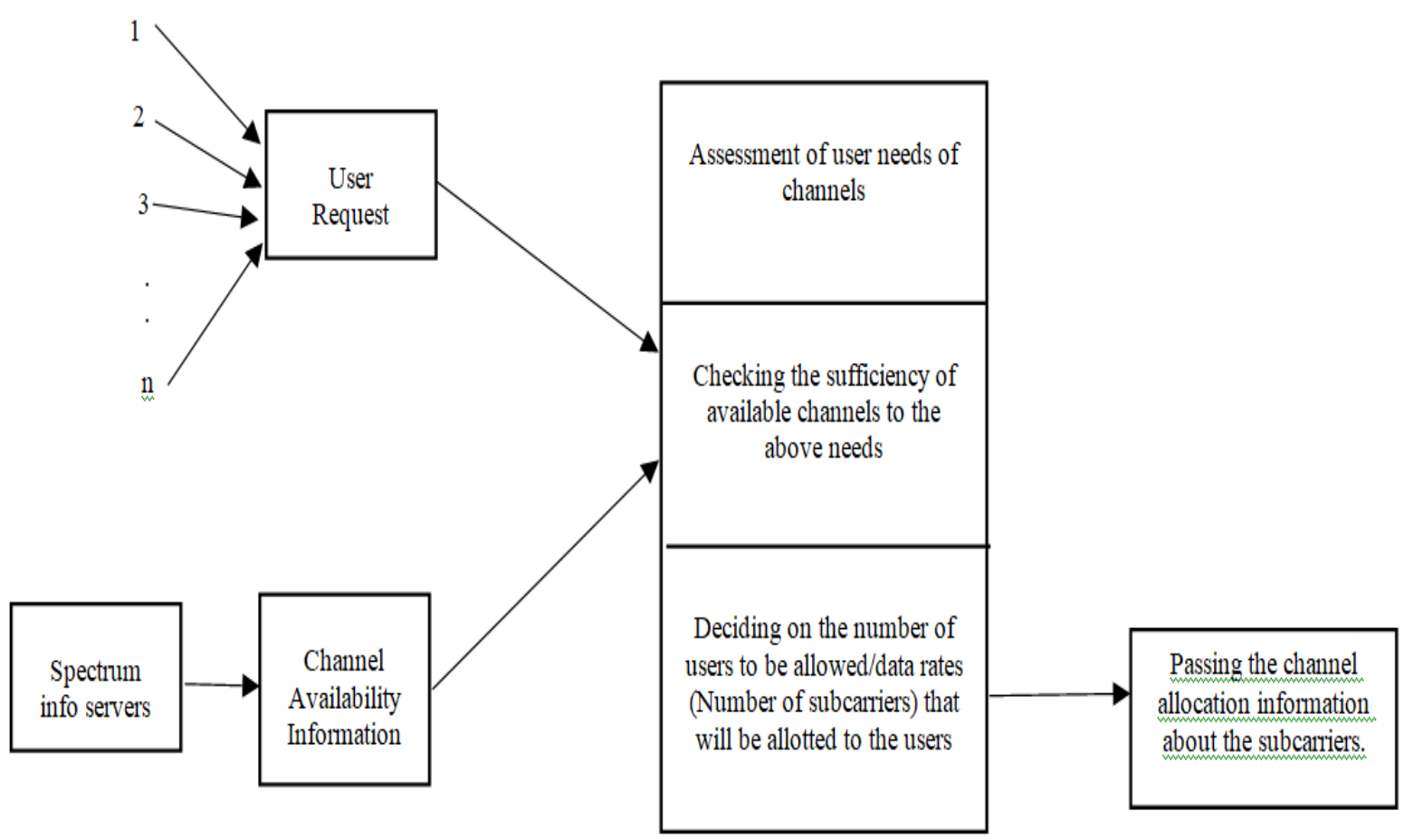

Figure 1: System model.

the optimized value. Section-4 describes the system model in terms of occupancy rate, data rate for the random occurrence of users, by adjusting the available frequencies based on the user request by using GLPK solver. Section-5 concludes the paper.

\section{ROLE OF COGNITIVE RADIO AND LTE}

\section{Role of Cognitive Radio:}

In cognitive radio networks, the unlicensed users that attempt to use the licensed spectrum during its unoccupied times, are called Secondary Users (SU's). There are four essential components involved in CR network implementation namely 1.Spectrum Sensing 2.Spectrum Decision 3.Spectrum sharing 4.Spectrum mobility. These components signify the life cycle of a cognitive radio network.

The first task is to perform sensing operation, where SU has to sense the channel before accessing it. After finding the spectrum holes also, SU has to sense the channel continuously because, if PU reappears then the SU has to vacate the channel instantly.

The second task is spectrum decision. In this task, after getting the information of vacant channels for SU's communication, an optimal assignment of these vacant channels to the requesting SUs has to be done. Here cooperation among SUs is essential for fair spectrum sharing.

The Third task is spectrum sharing, this task plays an active role in allocating appropriate frequency from the available vacant channels. Here several parameters have to be considered for spectrum sharing like energy consumption for low and high frequencies, propagation characteristics and so on. Here SU should know the PU's activity and has to act accordingly.

The fourth task is spectrum mobility. When SU is accessing the channel at a particular time, if PU reappears, the SU has to vacate the channel and switch over to another spectrum hole. This mechanism is called spectrum handoff. Here SU handoff should be done fast, because SU shouldn't cause any interference to the PU and QoS of SU's should not be degraded [11-14].

\section{LTE Frame Structure:}

LTE frame structure has two types, namely LTE FDD mode system and LTE TDD mode system. LTE FDD frame structure is a full-duplex system[15], i.e., both uplink and downlink transmissions can happen at the same time for different frequencies. In the LTE TDD frame structure, the half-duplex system is used, i.e., either uplink or downlink transmission occurs at one moment. In LTE, Downlink operation will be performed by Orthogonal FrequencyDivision Multiplexing (OFDM) and uplink operation by Single Carrier- Frequency Division Multiple Access (SCFDMA).

In LTE, each frame has a $10 \mathrm{~ms}$ duration. One Frame has 10 subframes, whereas each subframe has 2 Time slots, each Time slot having 7 OFDM symbols. A Resource Block (RB) has 12 subcarriers, and each subcarrier is spaced with $15 \mathrm{KHz}$, i.e., $(12 * 15 \mathrm{kHz}=180 \mathrm{kHz})$. An OFDM symbol is categorized in 3 ways, namely, BPSK Modulation (2 data bits), 16-QAM Modulation (4 data bits), 64-QAM Modulation (6 data bits). $100 \mathrm{RBs}$ are used for accessing 20 $\mathrm{MHz}$ channel.

In [16] conventional LTE frame structure consist minimum of 10 subframes, each subframe has $1 \mathrm{~ms}$ length and maximum of 40 subframes. By increasing the number of subframes that leads to the decrement of subframe length, which has less than $1 \mathrm{~ms}$ duration. 


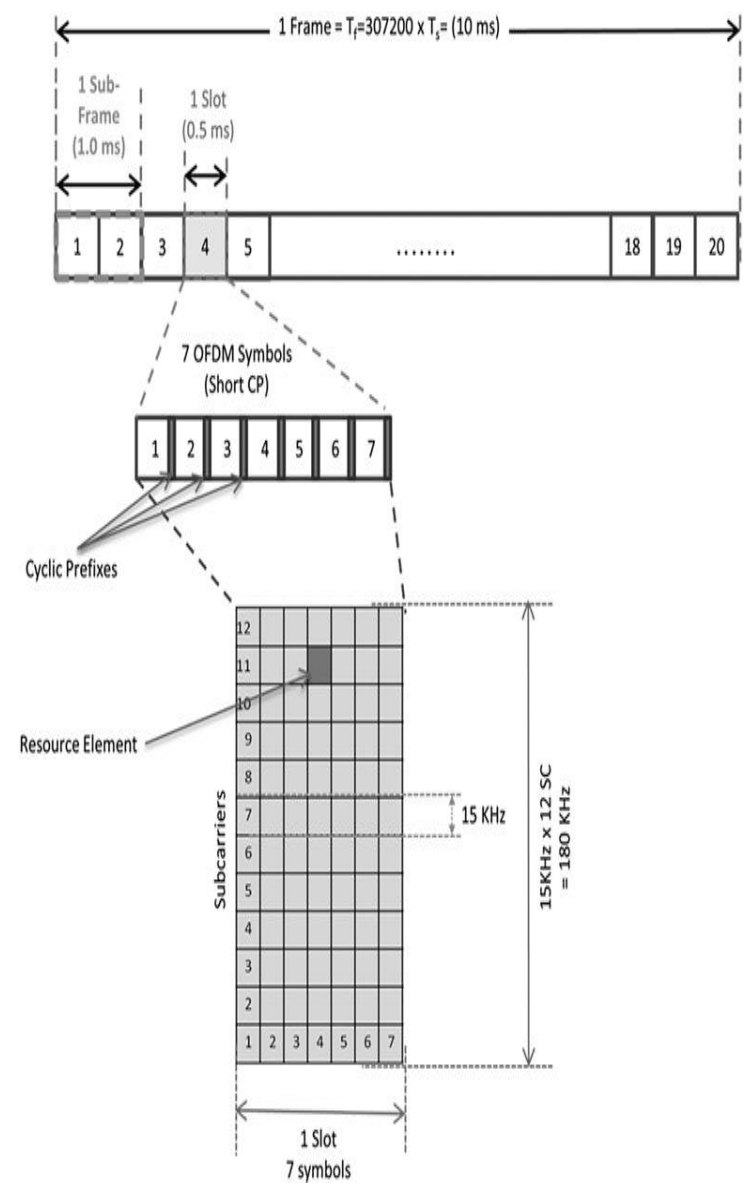

Figure 2: LTE frame structure [15]

\section{INTERVAL GRAPH}

The study of graphs is called graph theory, which is used to deal with mathematical structures in the real world modelling problems. A graph is made up of nodes or vertices and edges. Edge is nothing but the connecting line between the two nodes or vertices. A graph may be a directed or undirected. In directed graph, edges are directed from one node to another node. In the undirected graph, there is no direction to distinguish between the two nodes associated with the edge [17].

Interval graph is an undirected graph which shows the intersection intervals. These interval graphs are mostly used in scheduled mechanisms where the overlapping tasks shouldn't be performed i.e., each task should consist of precise start and end time. This interval graph is used to allot spectrum holes to requesting users. If more users are accessing the same channel at the same time interference will occur. To avoid that interference, different channels have to be assigned to different users or user has to access the channel in another precise start and end time.

Interval graph is represented in two forms. In the first form, task performance is represented with respect to time axis. In the second form, representation of shared and unshared edges of nodes is shown. Figure- 3 shows the sample task performance of 8 devices on time axis where overlapping tasks have existed. Figure 4 shows the Corresponding interval graph for Figure 3. Here when shared edge is there among the vertices it should assign a different color or different frequency to those vertices. Vertices with unshared edge can be assigned the same color or same frequency which was allocated previously.

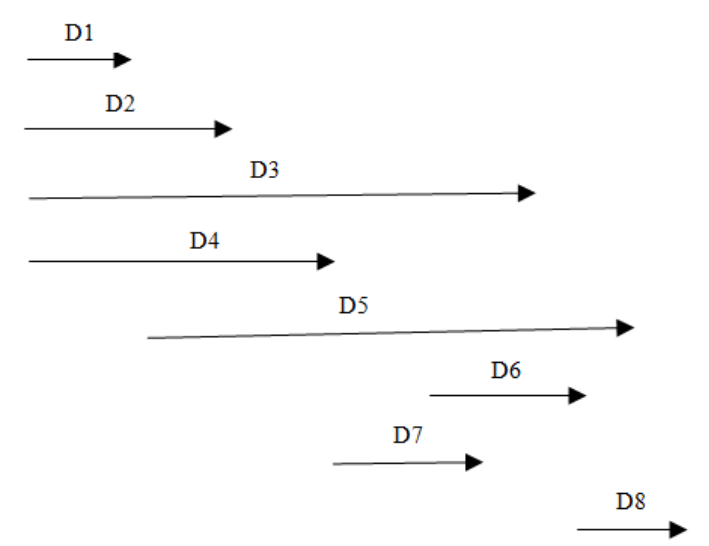

Figure 3: Task performance on time axis

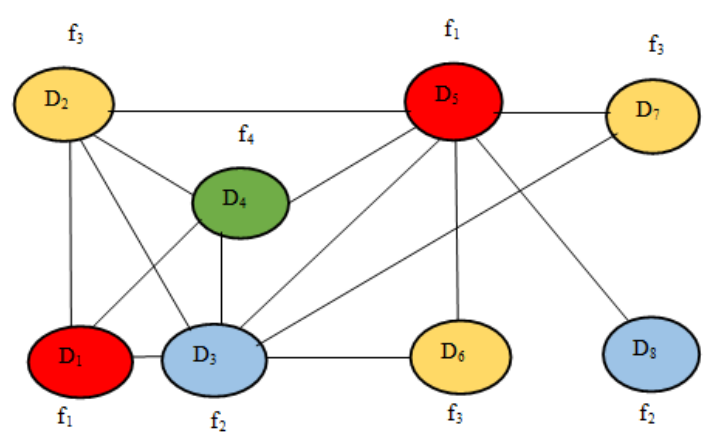

Figure 4: Interval graph for 8 Tasks

In figure 4 , objective of an interval graph is to minimize the number of frequencies/colors and the constraints here are: each and every node should be assigned only one frequency/color; No two shared adjacent node shouldn't assign same frequency/color; Reuse of frequency/color will happen for an unshared edge. From those constraints, the optimum value obtained was ' 4 ', i.e., 4 different frequencies/colors are assigned to perform the given task without any interference for 8 users.

To formulate the interval graph as an Integer Linear Programming problem, the objectives and constraints are defined as follows.

$$
\begin{gathered}
\text { objective } \quad \min \sum_{f \in U} y_{f} \\
\text { constraints } \sum_{f \in U} o_{V}^{f}=1 \forall v \in V \\
o_{V}^{f}+o_{\mathbb{V}^{f}}^{f} \leq y_{f} \forall\left(v_{v}, v^{v}\right) \in E, \forall S \in U \\
y_{f_{i}} \geq y_{f_{i}+1} \quad(i=1,2,3, \ldots \ldots \ldots \mid-1) \\
y_{f} \in\{0,1\} \forall S \in U \\
o_{V}^{f} \in\{0,1\} \forall v \in V, \forall S \in U
\end{gathered}
$$

Let " $U$ " be a set of "f" frequencies, where $U$ is Unlicensed frequencies $\mathrm{U}=\left\{\mathrm{f}_{1}, \mathrm{f}_{2}, \mathrm{f}_{3} \ldots \mathrm{f}_{\mathrm{lUI}}\right\} . O_{\mathrm{V}}^{f}$ and $y_{f}$ are binary variables. If $\mathrm{f}$ is assigned to the corresponding vertex $v, \quad O_{V}^{f}=1$, else $o_{\mathrm{V}}^{f}=0$. If $\mathrm{f}$ is used at least 
one time means $y_{f}=1$, else $y_{f}=0$. Equation (1) is the objective function which minimizes the required number of frequencies or colors. Equation (2) ensures that each frequency is assigned to the corresponding vertex. Equation (3) represents that different frequencies has to be allocated for two adjacent vertices to ensure that if same frequency is allocated to the adjacent vertex which leads to the interference. Equation (4) indicates that frequency has to be assigned in ascending order. Equation ( 5 \& 6) are last two constraints for assigning binary values to the given variables $y_{f}$ and $O_{D}^{f}$.

\section{System model for optimum frequency allocation and data rates using GLPK solver}

\section{System Model:}

A model that consists of 200 users with varying arrival distribution and data rate requirements is considered. The main motive of this model is to allocate available channels to the users according to their data rate requirements (i.e., available subcarriers) based on their occupancy and arrival rate. The proposed system consists of three models which are as follows:

The first model deals with Assessment of user needs of channels based on occupancy and arrival rates.

The second model is the extension of first model by considering different data rate requirements of different users randomly rather than considering the occupancy and arrival rates with a constant data rate of $1 \mathrm{Mbps}$ for every user.

Finally, the third model is a practical implementation of the above two scenarios in terms of subcarrier allocation in LTE technology.

GNU Linear Programming Kit (GLPK) is an opensource software to solve mathematical programs specifically LP (Linear programming) and MIP (Mixed Integer Programming).

In this simulation, arrival rate and occupancy of 200 users were considered randomly from $10 \%$ to $100 \%$ for 200-time slots, i.e., 200-time instances is simulated by using GLPK tool kit.

\section{Model 1:}

In this model, a scenario is considered where at a certain time instance when 2 or more users try to access the channel which leads to a collision. To overcome this case an interval graph concept is used to find an optimum value of frequency required for the successful transmission without collision. In figure 5, it is shown that for $10 \%$ user arrival rate, on an average users require 33 frequencies and for $20 \%$, the requirement is around 50. It continues linearly upto $90 \%$. Here an assessment of user needs of channels based on the varying arrival rate is observed. Depending on the available frequencies users may adjust their requirements. For example, if 100 frequencies are available, each user will set the requirement as $40 \%$ or below,

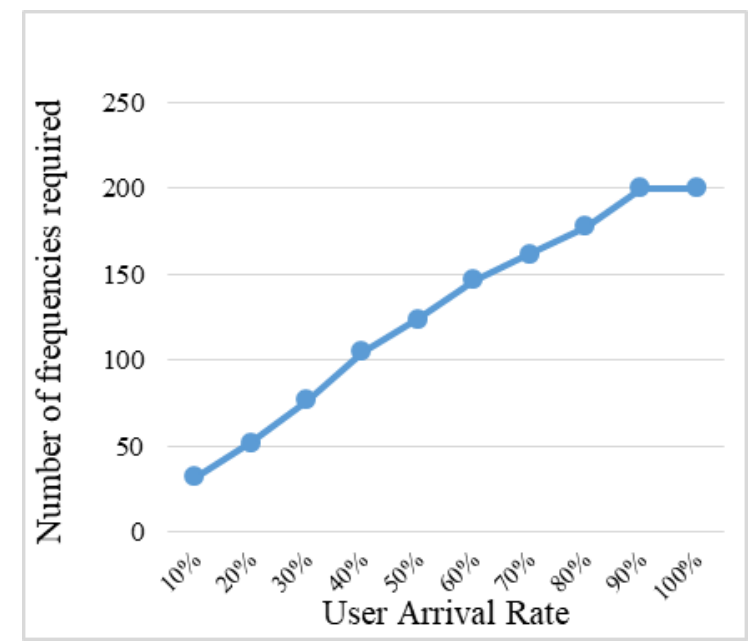

Figure 5: Assessment of user requirement of channel

Model 2:

In the first model, an assessment for the number of required frequencies based on user occupancy and arrival rates has been observed. In model 2, figure 6 shows the user arrival rate (vs) the number of frequency required. Not only the occupancy and arrival rate but also the data rate is considered here, for assessing the user needs of channels for required data rate. Whenever the user wants to access the channel, the requested data rate may not be the same every time. In this model users asking for different data rates is considered (i.e., from $1 \mathrm{Mbps}$ to $4 \mathrm{Mbps}$ ).

\section{Model 3:}

Model 3 describes the practical implementation of the above two cases. Here LTE technology with subcarriers concept is used. In the above mentioned models, one unit of frequency is treated as $1 \mathrm{Mbps}$ which consists of 66 subcarriers (12 Resource Blocks). So the problem is narrowed down to allocating the subcarriers for the users which are shown in figure 7 . In this diagram, available subcarriers are shown in horizontal line which intersects with the graph line and so on (\% of occupancy).

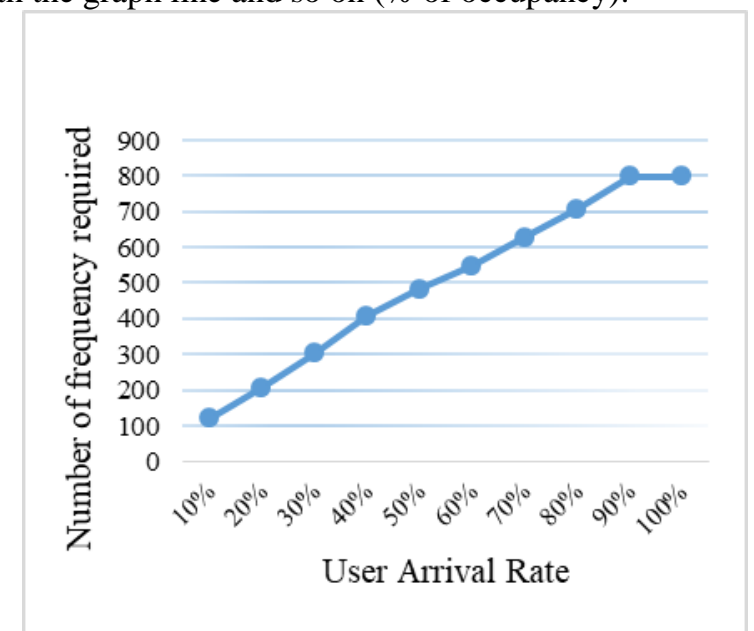

Figure 6: Assessment of user requirement of channel based on data rate 


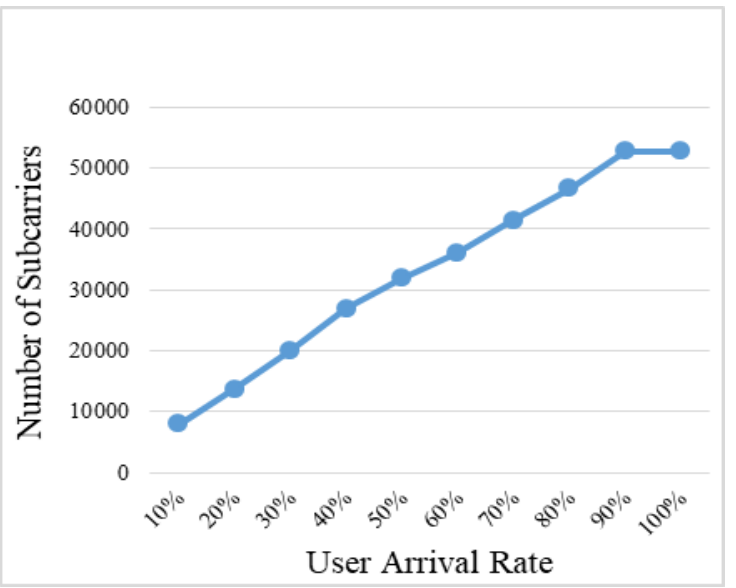

Figure 7: Allocation of Subcarriers to the users

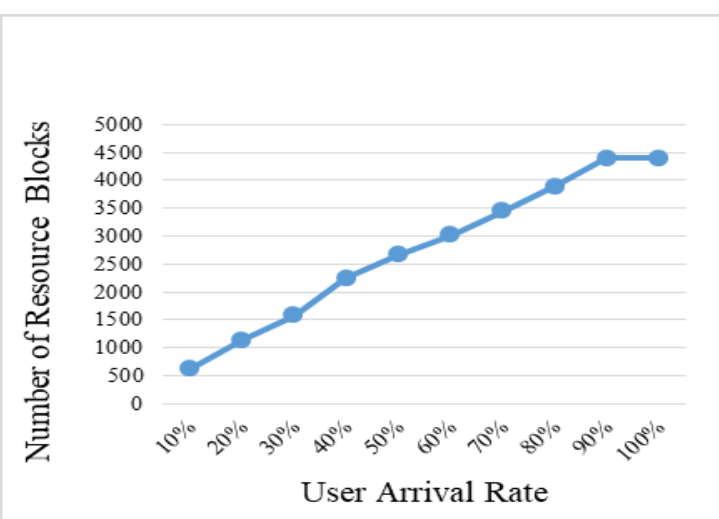

Figure 8: Allocation of Resource Blocks to the users

Table 1: Proposed models.

\begin{tabular}{|c|c|c|c|c|}
\hline \multirow[b]{2}{*}{$\frac{\frac{\text { User }}{\text { arrival }}}{\text {-rate }}$} & \multirow[b]{2}{*}{$\begin{array}{l}\text { Model- } \\
\underline{\mathbf{1}} \\
\text { (frequen } \\
\text { cy } \\
\text { required } \\
\text { ) }\end{array}$} & \multirow[b]{2}{*}{$\begin{array}{l}\text { Model-2 } \\
\text { (frequen } \\
\text { cy } \\
\text { required } \\
\text { basedon } \\
\text { data } \\
\text { rate) }\end{array}$} & \multicolumn{2}{|c|}{ Model-3 } \\
\hline & & & $\begin{array}{l}\text { (no.of } \\
\text { sub- } \\
\text { carriers } \\
\text { allocati } \\
\text { on to } \\
\text { users) }\end{array}$ & $\begin{array}{l}\text { (no.of } \\
\text { resour } \\
\text { ce } \\
\text { blocks } \\
\text { ) }\end{array}$ \\
\hline $10 \%$ & 32 & 120 & 7920 & 638 \\
\hline $20 \%$ & 52 & 208 & 13728 & 1144 \\
\hline $30 \%$ & 77 & 304 & 20064 & 1672 \\
\hline $40 \%$ & 105 & 408 & 26928 & 2244 \\
\hline $50 \%$ & 124 & 484 & 31944 & 2662 \\
\hline $60 \%$ & 147 & 548 & 36168 & 3014 \\
\hline $70 \%$ & 162 & 628 & 41448 & 3454 \\
\hline $80 \%$ & 178 & 708 & 46728 & 3894 \\
\hline $90 \%$ & 200 & 800 & 52800 & 4400 \\
\hline $100 \%$ & 200 & 800 & 52800 & 4400 \\
\hline
\end{tabular}

So users need to modify either their occupancy or data rate requirements. Table 1 shows the proposed models results for 3 models.

\section{CONCLUSION}

Due to the spectrum scarcity, effective utilization of available channels by users is one of important requirements. In this work, utilization of available TV white space frequencies is explored. At first, the number of channels (or) subcarriers required as per user demands is identified. If those many subcarriers are not available, then

downward data rate scaling that needs to be adopted by individual users is computed. While implementing this system, spectrum info of available channels will be obtained from spectrum info servers. Results indicate that multiple users can be offered frequency allocations as long as they are willing to adjust to share the available quantum of bandwidths. It can be extended to implement priority based allocations that offer higher quantum of allotments to realtime users.

\section{REFERENCES}

1. Mitola J, Maguire G.Q, Cognitive Radio: Making Software Radios More Personal, IEEE Personal Communications, pp. 13 - 18, August 1999.

2. Y. Huang, Y. Chen, Y. T. Hou, W. Lou and J. H. Reed, "Recent Advances of LTE/WiFi Coexistence in Unlicensed Spectrum," in IEEE Network, vol. 32, no. 2, pp. 107-113, March-April 2018.

3. G. Naik, J. Liu and J. J. Park, "Coexistence of Wireless Technologies in the $5 \mathrm{GHz}$ Bands: A Survey of Existing Solutions and a Roadmap for Future Research," in IEEE Communications Surveys \& Tutorials, vol. 20 , no. 3 , pp. $1777-1798$

4. R. Zhang, M. Wang, L. X. Cai, Z. Zheng, X. Shen and L. Xie, "LTEunlicensed: the future of spectrum aggregation for cellular networks," in IEEE Wireless Communications, vol. 22, no. 3, pp. 150-159, June 2015.

5. T. Baykas et al., "Developing a standard for TV white space coexistence: technical challenges and solution approaches," in IEEE Wireless Communications, vol. 19, no. 1, pp. 10-22, February 2012.

6. B. Gao, J. Park, Y. Yang and S. Roy, "A taxonomy of coexistence mechanisms for heterogeneous cognitive radio networks operating in TV white spaces," in IEEE Wireless Communications, vol. 19, no. 4, pp. 41-48, August 2012

7. G. P. Villardi, C. Sum, C. Sun, Y. Alemseged, Z. Lan and H. Harada, "Efficiency of dynamic frequency selection based coexistence mechanisms for tv white space enabled cognitive wireless access points," in IEEE Wireless Communications, vol. 19, no. 6, pp. 69-75, December 2012.

8. Meghalatha C.K, B. Seetha Ramanjaneyulu, R.S.Jagadeesh, Krishna Chaitanya .K, "Survey Of Research Issues And Standardization Efforts For The Utilization Of TV White Spaces"in proc. JARDCS , 2017, pp. 233-244.

9. Qian Zhang, Xinning Zhu, Leijia $\mathrm{Wu}$ and $\mathrm{K}$. Sandrasegaran, "A coloring-based resource allocation for OFDMA femtocell networks," 2013 IEEE Wireless Communications and Networking Conference (WCNC), Shanghai, 2013, pp. 673-678.

10. T. Qiu, W. Xu, Z. He, K. Niu and B. Tian, "Graph-based spectrum sharing for multiuser OFDM Cognitive Radio Networks," 2011 International Conference on Wireless Communications and Signal Processing (WCSP), Nanjing, 2011, pp. 1-5.

11. Mitola J, Cognitive Radio Architecture Evolution, Proceedings of IEEE, Vol.97, No.4, pp. 626-641, 2009

12. Mitola J, Maguire G.Q, Cognitive Radio: Making Software Radios More Personal, IEEE Personal Communications, pp. 13 - 18, August 1999

13. Joseph Mitola, Cognitive Radio: An Integrated Agent Architecture for Software Defined Radio, PhD dissertation at Royal Institute of Technology (KTH) Stockholm, Sweden, May 2000.

14. I. F. Akyildiz, W. Lee, M. C. Vuran and S. Mohanty, "A survey on spectrum management in cognitive radio networks," in IEEE Communications Magazine, vol. 46, no. 4, pp. 40-48, April 2008.

15. R. S. A. Raja Abdullah, A. A. Salah, A. Ismail, F. H. Hashim, N. E Abdul Rashid and N. H. Abdul Aziz, "Experimental investigation on target detection and tracking in passive radar using long-term evolution signal," in IET Radar, Sonar \& Navigation, vol. 10, no. 3, pp. 577-585, 32016.

16. M. Maule, D. Moltchanov, P. Kustarev, M. Komarov, S. Andreev and Y. Koucheryavy, "Delivering Fairness and QoS Guarantees for LTE/Wi-Fi Coexistence Under LAA Operation," in IEEE Access, vol 6, pp. 7359-7373, 2018.

17. A. Grzesik and H. Khachatrian, "On interval edge-colorings of complete tripartite graphs," Ninth International Conference on Computer Science and Information Technologies Revised Selected Papers, Yerevan, 2013, pp. 1-

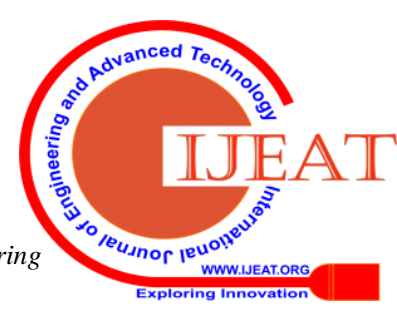

